Last, applying this strategy to human primary tumour samples specifically four B cell acute lymphoblastic leukaemias (B-ALLs) and three lung cancers - by subtracting mRNA sequences of TECs and mTECs from unrelated donors led to the identification of 2 mTSAs and 20 aeTSAs. One of the B-ALL aeTSAs originates from the 3 UTR of TCL1A, a gene associated with lymphoid malignancies. The next step would be to validate the immunogenicity of these TSAs in mice.

By identifying TSAs from noncoding regions, this study expands the number of targetable antigens that can be used for cancer vaccine development. Even more appealing as preferred targets, aeTSAs can be widely shared by multiple tumours (including those with low mutational burdens), meaning a single vaccine could be generated, unlike neoantigen-directed immunotherapy, which is likely to be personalized.

Anna Dart

ORIGINAL ARTICLE Laumont, C. M., Vincent, K. et al. Noncoding regions are the main source of targetable tumor-specific antigens. Sci. Transl Med. 10, eaau5516 (2018)

T cell receptor (TCR) and $B$ cell immunoglobulin repertoires from single cells of the same sample. This revealed that immune cell subsets, including exhausted T cells, and TCR clones were conserved between matched fresh tumours and organoids.

Last, to determine TIL functionality in these models, 20 further PDOs were generated from immunotherapyresponsive tumours, such as melanoma. Treatment with the PD1 antibody nivolumab resulted in induction of activation markers in expanding $\mathrm{CD}^{+} T$ cells and tumour cell killing in 6 of the 20 PDOs, indicative of response to immune checkpoint inhibition and consistent with clinical trial data.

This organoid method, preserving the immune contexture, has great promise for in vitro modelling of personalized immunotherapy.

\section{Anna Dart}

ORIGINAL ARTICLE Neal, J. T. et al. Organoid modeling of the tumor immune microenvironment. Cell 175, 1972-1988 (2018)

FURTHER READING Drost, J. \& Clevers, H.

Organoids in cancer research. Nat. Rev. Cancer

18, 407-418 (2018)

\title{
Trafficking signals for metastasis
}

Cytotoxic chemotherapy can effectively treat invasive breast cancer, but studies in mice have suggested that it can also have pro-metastatic effects. Keklikoglou et al. now show that, in mouse models of chemoresistant breast cancer, paclitaxel and doxorubicin trigger the production of tumour-derived extracellular vesicles (EVs) with pro-metastatic properties.

The authors first treated mammary tumour-bearing mice with paclitaxel, observing that it only modestly inhibited primary tumour growth. However, compared with vehicle, paclitaxel increased the incidence and size of lung metastases or the pulmonary seeding of mammary cancer cells, depending on the mouse model.

EVs can aid the metastasis of primary tumours by influencing cells associated with the pre-metastatic niche. Here, compared with controls, EVs isolated from 4T1 mammary tumour cells (4T1 cells) or murine mammary tumours following paclitaxel treatment increased the number of metastatic nodules in tumour-free mice challenged with metastasis-forming cancer cells. Thus, paclitaxel-induced EVs appear to promote breast cancer metastasis. As EVs derived from murine mammary tumours following doxorubicin treatment also enhanced lung metastasis in mice, this phenomenon is not paclitaxel-specific.

In assessing how chemotherapy-induced EVs promote breast cancer metastasis the authors first observed that paclitaxel increased the release of EVs from mouse and human cancer cell lines as well as the number of EVs in the blood of mice with 4T1 cell-derived tumours. Furthermore, proteomic analysis revealed that EVs derived from paclitaxel-treated 4T1 cells contained higher levels of the $\mathrm{Ca}^{2+}$-binding membrane-associated protein annexin A6 than EVs from vehicle-treated 4T1 cells. Paclitaxel and doxorubicin promoted the loading of annexin A6 into EVs from tumour-derived (but not non-transformed) cells, and annexin A6-deficient 4T1 cells did not promote lung colonization in mice in response to paclitaxel or doxorubicin. Thus, chemotherapyinduced $E V s$ require annexin A6 to promote lung metastasis.

CC-chemokine ligand 2 (CCL2) can reportedly enhance breast cancer metastasis that is assisted by lymphocyte antigen $6 \mathrm{C}(\mathrm{LY} 6 \mathrm{C})^{+} \mathrm{CC}$-chemokine receptor $2(\mathrm{CCR} 2)^{+}$monocytes. In line with this theory, treatment of tumour-bearing (but not

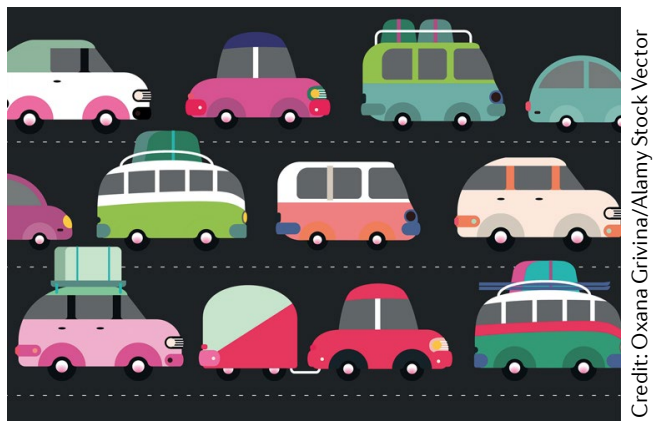

tumour-free) mice with paclitaxel increased the expression of CCL2 and the number of $\mathrm{LY}_{6 C^{+}}$ monocytes in the lungs. EVs isolated from paclitaxel-treated mice with tumours also increased the abundance of $\mathrm{LY}_{6 \mathrm{C}}{ }^{+} \mathrm{CCR2}{ }^{+}$ monocytes in the lungs of tumour-free mice. As paclitaxel-induced EVs did not promote pulmonary metastasis in Ccr2-knockout mice or increase the expression of $\mathrm{Ccl} 2$ or $\mathrm{Ccr} 2$, or the number of $\mathrm{LY}_{6 \mathrm{C}}+$ monocytes, in the lungs of annexin A6-null mice, annexin $\mathrm{A} 6$ may initiate metastasis by expanding $\mathrm{LY} 6 \mathrm{C}^{+} \mathrm{CCR} 2^{+}$ monocytes.

Finally, looking at the biology of cells in the lung pre-metastatic niche, the authors observed that paclitaxel promoted the internalization of $E V s$ by lung endothelial cells. By using murine endothelial bEnd. 3 cells, the authors found that annexin A6 was transferred from paclitaxel-induced EVs to $b$ End. 3 cells, where it co-localized with the nuclear factor- $\kappa B(N F-\kappa B$ ) subunit $\mathrm{p} 65$. NF- $\kappa B$ can activate the transcription of $C \mathrm{cl} 2$ and, here, paclitaxelinduced EVs increased NF- $\mathrm{KB}$ activity and $\mathrm{Ccl} 2$ expression in bEnd. 3 cells in an annexin A6-dependent manner.

Taking all of their data into account, the authors propose that chemotherapy-induced EVs transfer annexin A6 to pulmonary endothelial cells, where it promotes the upregulation of CCL2 and the accumulation of $\mathrm{LY}_{6 \mathrm{C}^{+}}$monocytes to allow tumours to colonize at metastatic sites. As annexin A6 levels were increased in the plasma of patients with breast cancer undergoing neoadjuvant chemotherapy, chemotherapyinduced EVs are potential biomarkers of pulmonary metastasis.

Katharine H. Wrighton

ORIGINAL ARTICLE Keklikoglou, I. et al. Chemotherapy elicits prometastatic extracellular vesicles in breast cancer models. Nat. Cell Biol. https://doi.org/10.1038/s41556-018-0256-3 (2018) 